the left lung became consolidated; on each of the four suc ceeding days there were fairly sudden and sharp collapses, from which she rallied after the administration of an enema with stimulant. Small doses of nux vomica and digitalis were also given by the mouth combined with an expectorant. After fourteen days, during which this infant, on account of her respiratory distress, had only the briefest snatches of sleep, she fell asleep for four hours. This was the turning point in her illness.

A similar tendency to recurrences of collapse was observed in the following case:

I saw a child, aged 16 months, son of a Brazilian naval officer, in consultation with the doctor of the Brazilian gunboat. Here, too, the lesion first affected the base of the right lung. Occasionally after the third day there would supervene collapse, during which the temperature would fall to $96^{\circ} \mathrm{F}$., and remain thus for an hour or two, during which the infant would be in a state of semi-stupor, quite indifferent to his surroundings or what was done to him. By means of hypodermic injections of caffeine at one time, and at another of ten drops or more of camphorated oil, coupled sometimes with a small enema of beef tea and brandy, the child would rally. In this patient, while the base of the right lung posteriorly was the part first affected, about the eighth day the lower and middle por tions of the right lung became affected and the patient passed through another attack of pnenmonia attended by similar collo and folls of temperature, but successully met by collaps of and falls of tomperate, but

The collapses in the pneumonia of young children resemble in some respects the depressions which occur in ptomaine poisoning attended by low temperature, only the semi-stupor is more marked.

Incomplete Recovery.

When pneumonia has not proved fatal, recovery may be complete or incomplete. Setting aside empyema as a sequel of pneumonia, I wish to draw attention to a pathological condition in the lungs-namely, the morbid anatomy of those unresolved pneumonias which end in fibrosis and chronic phthisis, conditions to which both Osler and Babcock in their books allude. The final conditions are somewhat analogous to those observed in lungs irritated by dust. The inhalation of dust causes pneumoconiosis. The effects of dust upon the lungs of dogs and rabbits in the early stages of an alveolitis are shown on the lantern slides by the proliferation of epithelial cells and the presence of large phagocytic cells which have englobed dust particles. The later stages are thicken. ing of the alveolar walls and increase of the fibro. connective tissue of the lang, especially around the small bronchi and the blood vessels, but in some of the other slides loops of capillaries can be seen bulging into the exudate within the alveoli, and inducing organization of the exudate. What would have happened eventually here had the patient lived would have been a marked increase of the fibrous tissue of the lungs, the fibrosis being the result of organization arising within the alveoli and the incorporation of the alveolar wall with it, whereas in pneumoconiosis the fibrosis rather proceeds from the wall towards the alveoli. As a consequence of organiza. tion of the exudate in unresolved pneumonias there take place thickening and hardening of the lung, followed by retraction and deformity of the chest wall.

Treatment.

I think the limits of time have already been exceeded. It is sufficient to say that I have not seen any striking result follow the use of pneumocoocal serum, nor, although often giving great relief to the breathing, can I recall a case of pneumonia having been saved by the inhalation of oxygen. Strychnine and digitalis have given me, on the whole, the best results; with pneumonia, as with many other illnesses, it is not the disease solely that requires to be treated, but the patient with the disesse.

1 Láncet, 1882, vol. i, p. 305. 2 General Bacteriology, 1909, p. 100.

A VERY successful meeting of the South-Eastern Division of the Medico-Psychological Association was held at Han well Asylum on April 26th, and was attended by a large number of the members of the Division. After the wards of the historic building had been inspected, the members were entertained to luncheon by. Dr. Percy J. Baily, the medical superintendent. Subsequently, at the meeting of the Division, Dr. Baily read a paper giving an account of the history of the institution, with special reference to the time when Conolly put into practice his humane ideas of non-restraint in the treatment of the insane. Papers were also read by Dr. Devine on Kraepelin's clinic, and by Dr. Phillips on the treatment of melancholia by the lactic acid bacillus.

\section{An Adotreg}

ON

\section{SONE POINTS CONCERNING PNEUMONIA} INI PNEUMOCOCCAL INFECTIONS

\section{IN CHILDH00D.* \\ BY}

T. R. C. WHIPHAM, M.D.Oxon;, M.R.C.P.LoND. PHYSICIAN TO THE EVELINA HOSPITAL FOR CHILDREN; ASBISTANT PHYSICIAN TO THE PRINCE OF WALES'S HOSPITAX.

I PROPOSE to deal with some points concerning pneumonia and pneumococcal infections in children as illustrated by some cases from the Evelina Hospital. While all will agree that bronchopneumonia is the more frequent type in early life, I wish to emphasize the fact that the lobar variety is commoner than is often supposed. Thus, among 150 of my own cases I found that 65 , or 43.3 per cent., were of the lobar type. The chief clinical distinctions between the two are these: (1) Lobar pneumonia is almost always primary, while bronchopneumonia is very frequently secondary; (2) the onset of lobar pneumonia is sudden and the symptoms are well marked, whereas bronchopneumonia begins insidiously as a rule; (3) the temperature in the lobar form tends to be more uniformly high and to end by crisis, whereas in the other the course of the fever is prolonged and irregular and rarely ends abruptly; (4) lobar pneumonia usually affects a localized area of one lung, at any rate to begin with, while bronchopneumonia. is bilateral and more extensive; (5) bronchopneumonia tends to occur at a slightly earlier age than lobar.

In lobar pneumonia the exciting cause is the pneumococcus, but in the bronchopneamonic form the infection is usually mixed-at all events, in the secondary casesthough it is true that in some of the primary cases the pneumococcus alone is found. Lobar pneumonia, according to my own experience, is most frequent during the second, third, and fourth years, 56.9 per cent. of my cases occurring during those years, whereas bronchopneumonia chiefly occurs during the first three years of life, as many as 70.6 per cent. of my cases being found in infants under. 4 years of age.

In lobar pneumonia one or other of the bases is most frequently attacked, most commonly the left, but in children it is not unusual for the apex of the lung to be affected. It is remarkable that if one apex is involvedprimarily, and not as the result of the extension of the disease from the lower lobes-it is practically always the right apex which is attacked.

The temperature in lobar pneumonia is usually high, and may remain for a time uniformly about $103^{\circ} \mathrm{F}$. or $104^{\circ} \mathrm{F}$., but, speaking generally, it shows more marked remissions in children than in adults. Occasionally, however, the temperature may be misleading in showing no marked rise. The crisis occurs, as in adalts, usually between the fifth and seventh days, but in some cases it may be delayed until towards the end of the second week or even later. In mild or abortive cases the fever may terminate on the third or fourth day. An ending by lysis is more common in children than in adults, and was found in 15.3 per cent. of my cases. Usually it is of short duration, and the temperature reaches the normal between the fifth and eleventh days. In bronchopneamonia, on the other hand, the temperature is generally lower and of an intermittent type; in some cases the rise may be only very slight-perhaps hot above $100^{\circ}$. The termination is by lysis - generally in two to four weeks-but in a number of cases the temperature does not remain normal until atter, perhaps, ten or eleven weeks, or even longer.

The diagnosis of pneumonia in the early stages is frequently a matter of difficulty if the pulmonary signs are not in evidence. Indeed, I am of opinion that in some instances the signs of consolidation may never appear. possibly owing to the lesion being deeply situated, and yet the case run a course consistent with a diagnosis of pneumonia. As an instance, I may quote the following cases :

* Delivered before the Tottenham Division of the British Medical Association 
CASE I.-Pneumonia without Characteristic Signs in the Chest : Recovery.

(Under the care of Dr. Charlton Briscoe.

William R., aged 2 years, had been perfectly well until two days before admission. There was an indeflnite history of a fall from his bed while playing, but the accident was not witnessed. Afterwards he was irritable and vomited, but took his nessed. Afterwards he was irritable and vomited, but took his food fairly well, and the vomiting did not continue. On admission he was quiet, the temperature was 99.6 F., but quickly furred, the pupils were equal and active, and there was no furred, the pupils were equal and active, and there was no photophobia. The head was not retracted, and there was no paresis in any of the muscles. The knee-jerks were present but diminished, the plantar reflex was flexor in type, and Kernig's sign was absent. No abnormal physical signs were found in the chest. The abdomen was flaccid, but both the liver and spleen were enlarged. On the following day the boy was sitting up playing, and the temperature had fallen to normal. The right cheek, however, was noticed to be flushed, and the palse continued quick, while the respiration-rate remained 40 . The percussion note at the right base was slightly
impaired, but the breath sounds were normal. At night the impaired, but the breath sounds were normal. At night the temperature rose to $102.2^{\circ}$, and the next morning the child hed two convulsions. Later on he was drowsy, and becsme deexeursions as high as $105.6^{\circ}$. The respirations varied between excursions high as $105.6^{\circ}$. The respirations varied between
-40 and 56 , but there were no further signs in the lungs. The blood showed a leucocytosis of 24,200 , and Widal's reaction was negative. Lambar puncture was performed, the fluid being clear and containing a very faint trace of albumen; only very few cells were found after centrifugalization. On the eighth day the temperature fell by crisis and remained normsl, the signs indieative of pneamonia were at any time found in the chest, and the child made a rapid recovery.

Cast I.-Pneumonia without Definite Physical Signs : Recovery.

Recovery.
Susan B., aged 9 years, was said to have been quite well until the morning of admission, when she "vomited bright blood." She had had an empyema in the left side of the chest five years previously. She was somewhat dazed ; the tongue was furred pulse 144, respirations 54. No abnormal physical signs in the chest conld be detected. She became very restless and delirious with occasional cough, laboured irregular breathing, and a bad pulse. During the night she vomited several times, necessitating rectal feeding. On the following morning the temperature was $102^{\circ}$, and the respirations were 56 . Slight dullness on percusfion was noticed in the right scapular region, but no other signs of consolidation. The heart was considerably dilated, and the pulse rapid and weak. The knee-jerks were absent, and the plantar reflex was extensor. The breath was very foul, and plantar reflex was extensor. The breath was very foul, and the. mouth and gums were dry. Widal's reaction was negative, and no fluid could be obtained by lumbar puncture. On the third day' a crisis occurred, and the respirations fell at the same time to 28 . The child improved rapidly afterwards, and the

One of the chief difficulties in early diagnosis is to distinguish between pneamonia and meningitis if cerebra signs predominate and pulmonary manifestations are not in evidence-if, that is, the case is one of what is termed the cerebral form of pneumonia. As an illustration of what I mean, I will quote another case of Dr. Briscoe's in which signs saggestive of meningitis were present.

CASE IIr.-Lobar Pneumonia: Signs suggestive of Meningitis : Empyema : Abdominal Tuberculosis : Almost Normal Tempera ture throughout: Death.

Sarah M., aged 1 year and 8 months, was first taken ill with diarrhoea nine days prior to admission. The diarrhoea ceased, but five days later the breathing became embarrassed, and the child had a constant dry cough. The face was pale, the eyes were sunken, and the breathing laboured. The temperature was normal, pulse weak and rapid, respirations 50 . The right side of the chest moved better than the left, which, if anything, was the greater in circumference. At the left base, posteriorly, there was an impaired percussion note, with patchy, tubular breathing, increased percussion note, with patchy, tubular tions; on the right side the note was resonant. The heart was not displa right side the note was resonant. The heart was not displaced, and the abdomen was normal. On the - morning after admission the temperature was $100.8^{\circ}$ - the was normal, and continued so, the physical signs in the lungs remaining the same for the next three days. The child, however, was slightly sick, and the head becam retracted, with the eyes fixed and staring, while the limbs showed marked muscular rigidity. At intervals the patien attered a shrill, high pitched cry. On the sixth day she was retching continually. The measurement of the chest was equal on the two sides, but on the right side near the angle of the scapula the note was impaired, and faint tubular breathing, with crepitations, could be heard when the child cried ; there was also an additional small patch at the free margin of the left was also an additional small patch at the free margin of the left vomiting and retching continued, and lumbar puncture yielded a drachm of perfectly clear fluid. The percussion note over the disappeared, and at the right base also the note became slightly deficient. On the tenth day death occurred. The temperature was practically normal throughout; on the second day it rose as stated, to $100.8^{\circ}$, and subsequently it once reached $100^{\circ}$, and on two other occasions $99^{\circ}$. At the necropsy there was some excess of fluid in the subarachnoid space, but no meningitis. The lungs showed acute bronchitis. There was a little pleurisy over the left lower lobe, which showed pneumonic consolidstion. On the right side there was a large empyema, with colle of was one early tuberculous ulcer, and the mesenteric glands were caseating.

In addition to the difficulty of diagnosis when many signs point to meningitis, this case emphasizes the fact that pneumonis, even when associated with empyema, may occar in children with scarcely any rise of temperature, and affords yet another example of how easily an empyema may be overlooked in the absence of any characteristic signs.

Meningitis may sometimes be a complication of pneumonia, and be due to an extension of the pneumococcal infection. In such crses the brain is found to be covered with a thick, greenish, puralent lymph, which is most extensive over the vertex and cerebellum, but frequently extend's to the base, and even to the medulla. The following is an instance of this complieation :

Case IV.-Whooping-cough: Bronchopneumonia: Pneumococcal Meningitis : Death.

Rosinin H., aged I year, was admitted under the care of my colleague, Dr. Nestor Tirard. For the previous two monkhs she had suffered from whooping-cough, bat for three days had been short of breath, with pain on coughing. She was a somewhat wasted baby, with typical whooping-cough. The face was flushed, and the veins in the neck were full. Signs of bronchoflushed, and the veins in the neck were full. Signs of bronchopneumonia were present all over the left lung. The temperature
was between $102^{\circ}$ and $103^{\circ}$, and continued irregular, but fell to was between $102^{\circ}$ and $103^{\circ}$, and continued irregular, but fell to
normal after nine days. Four days later it began to rise again normal after nine days. Four days later it began to rise again at night, and as the signs at the left base did not clear up, paracentesis was performed, when a large empyema was found and
evacuated. On the same day there was twitohing of the face, evacuated. On the same day there was twitohing of the face,
and the eyes deviated to the left. The child was continually sick, and general convulsions, with rigidity of the limbs; tol lowed. Death occurred on the following day. Post mortem the vertex of the brain was covered with thick greenish lymph, especially on the left side, and extending down deeply into the sulci. A amall amount of lymph was present over the cerebellam, but it did not extend to the spinal cord. In the chest there was adhesive recent pleurisy on the right side, and a large localized empyems cavity, which had been successfulty a large localized empyema cavity, which had been successfuliy emptied, on the left. The left lung. Was collapsed and studded with bronchopnetimonic areas; the right lower lobe was congested. A rabbit which wais inoculated with the cerebral exudate
died in twelve hours, its blood being found to be teeming with prieurrococei.

Occasionally, however, a septic meningitis or septic infection of the brain may follow an attack of pneumonia, the virulence of some septic focus being enhanced by the debility consequent on the pulmonary infection. The following is a case which serves to illpstrate this point.

CASE v.-Bronchopneumonia: Otitis Media: Septic Thrombosis of Cerebral Sinuses: Cerebral Abscesses: Death.

Albert C., aged 1 year, was admitted under my care. Eleven days previously he was taken suddenly ill with convulsions, and had a constant cough. He continued to be very fretful and restless, and steadily became worse. There was no history of otorrhoea or other illness. The child on admission was of an almost livid hue, the anterior fontanelle was depressed, and a frequent cough added greatly to the general respiratory embarrassment. The respiration rate was 72 , and the temperatare $103.6^{\circ}$. The chest, however, moved well, and there was no retraction of the intercostal spaces. On the left side these retraction of the intercostal spaces. On the lewer lobe, and were signs of a patchy consolidation in the lower lobe, and on the right there were a few moist sounds, especislly at the base. The knee-jerks were absent. The palcontinued irregular. On the fourth day the limbs on the right side suddenly became spastic and the head was retracted. 9 he spasm of the limbs passed off in a couple of hours, but the retraction continued and the child vomited. Repeated convulsions followed, starting on the left side, but later there was universal twitching of the whole body, and the head retraction became more marked. The child died four days after the onset of the cerebral signs. The meningeal vessels were found to be congested, with slight extravasation over the vertex on the right side. A septic thrombus was present in the spperior right side. A sep, and the veins leading to it were thrombosed. In the right lateral sinus was a recent thrombus, and in the In the right lateral sinus was a recent thrombus, and in the straight sinus was a white, slightly adherent clot. A small abscess cavity was present in the left occipital thick yeilow pus, and communicating with the lateral sinus on that side. Both 
middle ears contained pus. In the lungs there was acute purulent bronchitis, and the left lower lobe was solid with bronchopneumonia, while the right showed a large area of collepse.

In this case there was evidently a chronic otitis, which had been stimulated into activity as a result of the bronchopneumonia, and had caused a septic infection of the brain and cerebral sinuses.

Although various pneumococcal infections are liable to follow a recent attack of pneumonia, it does not always happen that there is a history of a previous inflammation of the lung. The different serous membranes are at times attacked, apparently primarily. Thus, in another of my cases pneumococcal meningitis associated with cerebral abscesses occurred in an apparently healthy child.

CASE vi.-Pneumococcal Meningitis: Cerebral Abscesses : Death.

Cyril E., aged 4 years, was suddenly taken ill with vomiting one night. This was followed by a fit in which all his limbs twitched. He became feverish and lost his appetite, and on the third day was brought to hospital. He had previously had third day was brought to hospital. He had previously had chicken-pox, measles, whooping-cough, and scarlet fever, but was. however, a history of an alleged fall on the head about a was, however, a history of an alleged fall on the head about a
week before admission. He had an apathetic, vacant look, and was drowsy, resenting any interference. The eyes tended to deviate to the right, and the right arm and leg seemed weak. There were no abnormal signs in the chest or abdomen. The knee-jerks were absent and both plantar reflexes were of the extensor type. Sensation seemed unsffected and there were no changes in the optic discs. The temperature was slightly raised, the pulse-rate 110 and the respirstions 40 . On the sixth day of his illness he had twitchings on the right side, and was subsequently sick. On the eighth day another fit occurred, but this time on the left side, the face being also affected, and the eyes turned to the left. The fit was easily checked by chloroform, but paresis of the left arm and leg resulted. On the following day the right arm was found to be paralysed. This subsequently recovered to a certain extent, but the vomiting subsequently recovered to a certain extent, 'but the vomiting becsme more frequent. Lumbar puncture, however, failed to yield any cerebro-spinal fluid. The temperature kept for the most part between $99^{\circ}$ and $100^{\circ}$ until the fourth week, when it suddenly began to rise, with the development of an attack of siarlet fever, the origin of which was obscure; possibly it had been conveyed by the patient's friends. The child was sent to a fever hospital and returned in about a month. He was then pale and drowgy with sunken eyes, and showed paralysis of the left arm and leg. The temperature was normal and the pulse rather slow. He was frequently sick, and the left eye became prominent. Ten days after readmission the temperature began to rise again irregularly to $100.6^{\circ}$. A diagnosis of cerebral abscess being made, Mr. Scott trephined the patient, but failed absess being made, Mr. Scott trephined the patient, but failed to discover any pus. The child gradually sank and died a little ever two months after being frst taken ill. At the post-mortem examination the cerebral convolutions were flattened and the
base of the brain was covered with greenish pus. In the left temporo-sphenoidal and left frontal lobes were abscesses each of the size of a large walnut. Pas was present in the lateral ventricles especially on the left side, where the anterior corna was considerably dilated. The ears were normal, and no changes of importance were found in any of the viscers. Cultivations from the pus grew the pneumococcus in pure culture.

Again, a pneumococcal infection of a joint is occasionally met with. The following case, which was recently under the care of my colleague, Mr. Mollison, affords another instance of a serous membrane being primarily affected.

CaSE VIr.-Pneumococcal Arthritis : Operation : Recovery.

Elsie L., aged 1 year and 3 montbs, was admitted with a six weeks' history of swelling of the right knee, which gradually bacame painful. The child was said to have been unwell six oi eight weeks previously, but there was no history pointing to pneumonia. The joint was swollen, and was at once explored, some turbid synovial fluid being withdrawn, which on cultivation yielded a growth of pneumococcus. The joint was opened, and turbid fluid containing flakes of $19 \mathrm{mph}$ was evacuated. The temperature was normal on admission, and only slightly raised for a couple of days after the operation. The child made a good recovery.

The origin of infection in such cases where there is no antecedent pneumonia is obscure. The organism must either be in the system and be stimulated into activity by some debilitating disease or injury, or the weakening effects of some illness may predispose the patient to an invasion by the pneumococcus. An instance of a general invasion by this organism as the result of severe illness is the following. The case is remarkable in that there were three distinct morbid processes going on at the same time, which consequently rendered a diagnosis extremely difficult.
CASE VIII.-Pericarditis: Acute Rheumatism: Tubercle of the Lung: Pneumococcal Septicaemia: Death.

George N., aged 4t years, was under the care of Dr. Fenwick. The patient had had measles about a year before. He was first taken ill with "pains in the stomach" nine days previous toadmission, and had been under a doctor with a constant temperature since. The boy seemed in pain, and screamed The area of cardiac dullness extended from the nipple line on the left to $\frac{1}{2}$ in. beyond the sternum on the right. The heart sounds were normal, but well-marked periardial friction was audible all over the precordium. The percussion note over the base of the left lung was impaired, and the breathing at the left apex was deficient. The abdomen was rigid and painful in the median line, but nothing abnormal could be felt. The temperature was $103.8^{\circ}$, pulse 112 , respirations 64. The temperature continued hectic for the first three days, and then fell somewhat, though keeping irregular between $96^{\circ}$ and $100.5^{\circ}$. The friction became less marked during this. time, and finally disappeared on the ninth day after admission. The patient increased slightly in weight and showed someimprovement, but a fortnight later the left knee became. swollen and painful, and there was some effusion into the joint without any additional rise of temperature. The joint. subsided under the administration of aspirin, but a week later the right knee was similarly affected, the temperature two the right knee was similarly affected, the temperature two days previously having risen temporarily to $103.6^{\circ}$. Sodium another week the pain recurred and the ankles and wrists were affected, all the joints being swollen. The heart's apex was now. $\frac{1}{2}$ in. outside the left nipple Jine. The salicylate was increasea and the true salt substitated with good effect; the temperature gradually fell, and kept chiefly subnormal for about two weeks. It then began to shoot up again, chiefly in the mornings, and the pain returned in several of the joints. The spleen became. enlarged and a transient bright red rash appeared chiefly on the limbs. The temperature again subsided, though it was not quite steady, but in anotber week again began to show exacerbations to over $104^{\circ}$ in the early morning, one of which was ccompanied by a rigor. These rises continued for nearly a month with the exception of two periods of three or four days each when the temperature was almost normal. The erythematons rash again appeared, but faded after three days, and the pleen increased in size. Ophthalmoscopic examination showed nothing abnormal, and a blood count showed no leacocytosis, the white corpuscles numbering 9,600 per c.mm., while the red were $4,720,000$ and the haemoglobin 65 per cent. A differential count of the leucocytes showed that the various forms were present in the normal proportions. On three different occasions. caltivations were made from the blood, but no growth was obtained except on one when a sarcina appeared, which was probably due to a contamination from the skin. Between the: rises of temperature the patient seemed quite well, and it is noticeable that his body-weight remained gteady though it dia not increase. With the fever he complained of headache especially in the occipital region. Two further rigors then accompanied rises of temperature, and the patient was not $s c$ accompanied rises of temperature, and the patient was not ser. well. He was rather cyanosed, and was delirious at times, and painful. The temperature became more uniformly raised, and as the signs in the left lung still continued the pleura was ex plored, but without result. The temperature again fell, but another rigor soon occurred. The feet and hands became swollen and the boy was a good deal troubled with cough. He was sick at times and gradually went downhill, the temperature being continuously very irregular at the last. He finally died, three and a half months after his admission. At the posts mortem examination the superficial vessels of the brain were found congested, but there was no meningitis. The pericardium. was firmly adherent; the heart and valves were normal. Both pleurae showed recent adhesions. Throughout the left lang pleurae showed recent adhesions. Throughout the left lung were small areas of caseation and larger areas of consolidation; the right was congested, but otherwise normal. The liver was congested and slightly fatty; the spleen was large and soft and the kidneys were cloudy. The pnenmococcus was found in the blood within the heart, and a rabbit injected with the serum
died suddenly in a couple of dass with abundant ovidence of died suddenly in a coupl

The simultaneous occurrence of rheumatism, tuberculosis, and pneumococcal infection is unusual. The tabercle was probably of some little standing, and the debilitating influence of this and the superadded rheumatism rendered the child susceptible to a virulent invasion by the pneumococcus.

I now tarn to some abdominal complications of pneamonia. A rare complication is acute nephritis. Albs. minuria is, of course, common enough during the febrile stage, but evidence of an actual parenchymatous nephritis is sufficiently uncommon to jastify my detailing this case :

Case IX.-Lobar Pneumonia : Acute Nephritis : Recovery. Matilda O., aged 4 years, a fat, healthy-looking child, had had a severe "cold" for a fortnight, but suddenly began to getworse three days before admission. She had been delirious had complained of general and abdominal pain, and had vomited. When seen, she was drowsy and very tremulous; shetant tabular breathing was heard at the right bsse. The, 
abdomen was very tender, but the tenderness could not be localized, and nothing abnormal could be felt. The tempers ture varied between $99.5^{\circ}$ and $104.6^{\circ}$, and after three days considerable dilatation of the heart was noticed. The urine was tested on admission, and on subsequent occasions, but no albu mon was found. On the tenth day, however, blood was found to be present, and the spleen at the same time could be easily lelt. The blood continued to be present, and the albumen inereased to 23 per cent. (Esbach), while granular casts were found on microscopical examination. The temperature was irregular up to $102^{\circ}$ for another ten days, when it sank to normal, and the renal and pulmonary signs, the latter of which showed considerable delay in clearing up, subsided at the same time. The patient made a good recovery.

Pneumococcal peritonitis is a severe, and often a fatal, condition. It may occur as a complication of pneumonia or it may have a primary origin, and perhaps be followed by pneumonia, as happened in the second of the two cases which I shall now quote. In the majority of cases occurring in childhood this condition is a primary one, and is generally of the encysted form-that is, the purulent exudate is localized, and is usually found in the lower part of the abdomen. A secondary peritonitis, on the other hand, is, as a rule, diffuse, and is less common. The first of the two peritonitis cases which I have to bring to your notice illustrates the involvement of the pericardium and plurae, in addition to the peritoneum, secondarily to an attack of pneumonia.

CaSE X.-Double Lobar Pneumonia: Double Empyema Pyopericarditis : Peritonitis : Death.

Ethel W., aged 41 years, came of a family in which there was -a history of tubercle and chest troubles. She had had pneumonia following measles two years previously. About five weeks before admission to hospital she passed through an attack of pneumonia, which involved both lungs, the crisis - occurring on the fifth day. The signs in the lungs, however, did not clear up, dullness persisting at both bases and moist sounds being audible on both sides of the chest. Towards the -end of the second week it was noticed that her abdomen began to swell, and it gradually became larger. The breathing became more embarrassed and the child lost flesh. On admis. sion the temperature was $100.2^{\circ}$, it having been previous to this practically normal after the initial fever, and the pulse was 140 . 'practically normal after the initial fever, and the pulge was 140 . :sounds were best heard over the fourth costal cartilage. There :sounds were best heard over the fourth costal cartilage. There was dullness over both lower lobes of the lungs with greatly
diminished breath sounds and absence of vocal fremitus. The diminished breath sounds and absence of vocal fremitus. The fuid. Paracentesis of both sides of the chest was performed pus being withdrawn from the left, but not from the right. On the operating table a little more pus was withdrawn from the left pleura by means of a needle, but on resecting part of a rib no further collection could be found. The patient was very -collapsed afterwards, and the cyanosis increased. On the assumption that the respiratory difficulty might have been caused by the pressure of the fluid within the abdomen, the peritoneum was tapped some twenty-four hours later and peritoneum was tapped some twenty-four hours later and abdomen was opened in the middle line below the umbilicus abdomen was opened in the middle line below the umbilicus,
when 3 to 4 pints of thick yellowish-green pus were evacuated, when 3 to 4 pints of thick yellowish-green pus were evacuated, ever, never rallied, and death occurred suddenly two days later. The temperature, except on admission, never rose above $99.6^{\circ}$ At the post-mortem examination there was no meningitis. The pericardium was distended by $16 \mathrm{oz}$. of thick greenish pus. There was generalized pleurisy over both lungs and a small empyema on either side posteriorly. The lungs were oedems'tous, and the left lower lobe was very congested, but no signs of consolidation remained. The peritoneum was uniformly covered -by a thick layer of lymph, which had caused numerous adhesions, -shutting off here and there small isolated collections of pus. The lymphatic glands were not enlarged. Caltures of the pus from the pleurae, pericardium, and peritoneum yielded pure cultures of pneumococcus.

The case seems to have been one of primary pneumonia with secondary infection of the other serous membranes. There was no evidence, however, to show that the peritoneum had become infected by direct extension from the pleurae. The diaphragm did not show any signs of disease, and the pelvic peritoneum was more extensively involved than the upper. On the otber hand, there was no evidence that the peritonitis was due to an infection from the alimentary tract or from the pelvic organs, which were normal. The pyopericarditis was entirely undiagnosed. The other case is an example of a primary infection of the peritoneum :

Case xr.-Pneumococcic Peritonitis : Secondary Pneumonia Death.2

Edith H., aged 5 years, when 15 months of age had an attack of "inflammation of the bowels." Since then she had :attack of "inflammation of the bowels." Since then she had abdominal pain, worse at times after food. There had been no vomiting, and the bowels were regular. Five days before admis sion she woke with severe pain in the abdomen, and vomited, the temperature being $103^{\circ}$. The pain continued, and diarrhoes persisted after a dose of castor oil, but the vomiting ceased. The abdomen became distended and somewhat rigid, especially in the lower part, and the temperature continued high. When seen the patient's aspect suggested pneumonia rather than peritonitis, the face being flushed and the respirations quick ened. The abdomen was distended and only slightly tender; it was resonant, and afforded no evidence of free fluid. There were signs of consolidation over the lower lobe of the left lung, and some crepitations and coarse râles at the right base. A blood count showed a leucocytosis of 15,000. Laparotomy was deferred, 3 the case did not seem to be one of septic perideferred, as the case did not seem to be one of septic peritonitis, and the presence of pneumonia suggested that it was a pneumococcal infection. The dullness on the right side of the chest became more marked and more extensive, so that fluid was
suspected. Exploration, however, was negative. The abdcsuspected. Exploration, however, was negative. The abdcmen continued distended, and some diarrhoea with very
offensive stools followed. Antipneumonic serum was given on two occasions without any noticeable benefit. The temperature then fell, and the lungs showed signs of resolving, the abdomen at the same time becoming much less distended and the diarrhoea ceasing. Some induration was felt in the hypogastrium, but operation was further deferred in order that the child might be in a better condition as the lungs improved. A sudden change, however, occurred. The temperature rose and the abdomen rapidly became enormously distended, with signs of intestinal perforation. Laparotomy was immediately performed in the middle line of the hypogastrinm, when free performed in the midale line of the hypogastrinm, when free gas and $8 \mathrm{oz}$. to $10 \mathrm{oz}$. of thick greenish fluid containing flakes of lymph were evacuated. The intestines were extensively matted together, so that it was impossible to find a perforation. Many incisions for the purpose of irrigation and drainage were consequently made, but the child, as was onj to be expected, died three days later from septic intoxication. At the necropsy general adhesive peritonitis and small collections and the diaphragm was a large abscess entirely shut off from the general peritoneal cavity. No perforation could be found. Both lungs showed areas of collapse and slight pneumonic consolidation. Films from the pus within the abdomen showed the presence of the pneumococcus.

In this case the abdominal infection seemed to be primary, the pulmonary signs not appearing until the third day. As no perforation could be discovered, it is possible that gas-forming organisms gained access to the peritoneal cavity through a damaged intestinal wall.

The cases related cover a wide area, and serve as illustrations of the difficulties at times attending the diagnosis of pneumonia and its complications in child hood, as well as reminders of the extensive ravages which the pneumococcus may bring about.

1 Clogg, British Journal of Children's Diseases, 1907, vol. iv, p. 491 2 Cloge,

\section{HOSPITAL INFECTION OF TUBERCULOSIS}

As Exemplified by the Records of the Resident Staff of the Modnt Vernon Hospital for Consomption and Diseases of the Chest for the past Fifteen Years

By J. EDWARD SQUIRE, C.B., M.D., F.R.C.P., SENIOR PHYSICIAN TO THE HOSPITAL.

A RECRN $\mathrm{T}$ contribution ' on the infection of consumption by Dr. Theodore Williams raises again the question of hospital infection, and refers to the original figures from the Brompton Hospital on this subject which have been so widely quoted as evidence of the comparative immunity from tuberculous infection of the resident medical and nursing staffs of special chest hospitals.

These figures, and others which have been brought forward, appear, however, to be compiled from the results of inquiries into the health of the former residents and nurses rather than from any actual medical examination of the individuals concerned, and are thus inconclusive. In one case-that of Victoria Park, given by Dr. Andrew in his Lumleian Lectures ${ }^{2}$ - the information appears to have been simply obtained from the secretary of the hospital.

As a further contribution to this question, I propose to give some figures from the Mount Vernon Hospital which have at least the advantage of being compiled from actual examination of the individnals. As Dr. Williams's latest article gives particulars of the resident medical officers, I have added to my statistics particulars of the resident *A paper read before the Medical Section of the Royal Society of
Medicine, April $26 \mathrm{th}, 1910$. 